\title{
What is the right place for atypical exemplars? Commentary: The right hemisphere contribution to semantic categorization: a TMS study
}

\author{
Maria Montefinese $^{1}$, Marco Ciavarro ${ }^{2}$ and Ettore Ambrosini ${ }^{1 *}$ \\ ${ }^{1}$ Department of Neuroscience, University of Padova, Padova, Italy, ${ }^{2}$ IRCCS Neuromed Pozzilli, Pozzilli, Italy
}

Keywords: concept typicality, semantic categorization, hemispheric lateralization, rTMS, coarse activation hypothesis

\section{A commentary on}

The right hemisphere contribution to semantic categorization: a TMS study

by Passeri, A., Capotosto, P., and Di Matteo, R. (2015). Cortex 64, 318-326. doi: 10.1016/j.cortex.2014.11.014

OPEN ACCESS

Edited by:

Andriy Myachykov,

Northumbria University, UK

Reviewed by:

Hidenao Fukuyama,

Kyoto University, Japan

Chris McNorgan,

University at Buffalo, The State

University of New York, USA

*Correspondence:

Ettore Ambrosini,

ettore.ambrosini@gmail.com

Specialty section:

This article was submitted to

Cognition, a section of the journal

Frontiers in Psychology

Received: 25 July 2015 Accepted: 24 August 2015 Published: 08 September 2015

Citation:

Montefinese $M$, Ciavarro $M$ and Ambrosini $E$ (2015) What is the right place for atypical exemplars? Commentary: The right hemisphere

contribution to semantic categorization: a TMS study.

Front. Psychol. 6:1349

doi: 10.3389/fpsyg.2015.01349
Categorization helps organizing our world knowledge by classifying exemplar concepts into categories. The degree to which an exemplar is representative of its category is called typicality (Rosch and Mervis, 1975). Passeri et al. (2015) made the first attempt to assess its effect on semantic representations in Wernicke's area (IW) and its right homolog (rW) by interfering on their online activity with repetitive Transcranial Magnetic Stimulation (rTMS) while participants categorized typical and atypical exemplars. Responses to typical and atypical concepts were delayed, respectively, by rTMS over both areas and rW only, supporting the "coarse activation hypothesis" (Jung-Beeman, 2005), according to which semantic processing is coarser in right (RH) than left (LH) hemisphere. Despite the authors' effort to clarify hemispheric contribution to semantic categorization, their contribution is hindered by some theoretical/methodological limitations that are worth discussing.

Firstly, the authors interchangeably referred to contrasting semantic memory (SM) theories, making it difficult to conciliate their hypothesis with them. For example, in saying that "Typicality effect can be due to [...] features shared or cooccuring between members of a category" (p. 319), they relied on feature-based semantic theories (e.g., McRae et al., 1997; Montefinese et al., 2014), which suppose a distributed network of featural representations in which typicality reflects featural intercorrelation (McRae et al., 1999; Montefinese et al., 2015). Contrastingly, in saying that "Typicality effect can be due to the number, proximity or binding of features" (p. 319), they relied on the spreading activation theory (Collins and Loftus, 1975), which supposes a somewhat hierarchical SM structure in which typicality -so-called criteriality- reflects the weight of links between basic-level and superordinate nodes. However, the authors left this dilemma open by seeking support in the Beeman's coarse activation hypothesis and sustaining that hemispheric differences "can be more simply traced to the different characteristics of the semantic fields and such an explanation lends itself to account for the effects regardless of any theoretical approach to the concepts organization" (p. 323). Besides, we would underline that, as far as we know, the original biological model (Jung-Beeman, 2005) was not integrated with connectionist models of the SM structure. Regrettably, thus, the authors missed the opportunity to clarify the cognitive processes implicated and did not provide the reader with adequate information to understand their (implicit) assumption that the semantic field is comparable to the SM structure and how to 
integrate the typicality (not to say the criteriality or feature correlation) within Beeman's theory. More importantly, Passeri and colleagues hypothesized that "processing of atypical members, whose features are more weakly or remotely correlated with other category members and that are more distant from the category, specifically involves the RH” (p. 320). However, they did not manipulate either criteriality or feature correlation but adopted concept-related typicality ratings and production frequency. Moving from the Passeri et al.'s findings, future works should try to overcome this limitation by assessing the effect of these fine-grained measures, as well as that of concept familiarity, which is known to affect several semantic tasks including categorization tasks (Glass and Meany, 1978)- and shares variance with typicality (Montefinese et al., 2013).

Another critical point concerns the authors' hypotheses about $\mathrm{RH}$ role in categorization. Indeed, it is unclear how they infer that $\mathrm{rW}$-rTMS should cause a response times (RTs) slowdown in atypical concepts categorization (p. 320). Rather, as suggested by the literature cited (Harpaz et al., 2009), one could make different predictions. Indeed, Harpaz et al. (2009), which also aimed to verify the Beeman's theory (Jung-Beeman, 2005), found more accurate responses and higher sensitivity to subordinate meaning blocks following RH-rTMS in a semantic decision task. Thus, even if Passeri and colleagues derived the trial timeline and rTMS protocol from Harpaz et al. (2009), they found quite different results. Nonetheless, the interpretation provided by the authors for this apparent incongruence was not fully satisfactory. Indeed, by relying on the fact that Harpaz et al. used a block design, they called into question putative "expectation effects on the task that increased the accuracy but nullified difference in RTs" (p. 324). However, Harpaz et al. found hemisphere-dependent effects on accuracy/sensitivity, with a clear double dissociation of the rTMS effect, and thus it is unclear how the rTMS could have modulated an unspecific expectation effect in a hemisphere-dependent way. Furthermore, another rTMS study showed that, in a picture-word matching task, only IW-rTMS delayed participants' response

\section{References}

Collins, A. M., and Loftus, E. F. (1975). A spreading-activation theory of semantic processing. Psychol. Rev. 82:407. doi: 10.1037/0033-295X.82.6.407

Fuggetta, G., Rizzo, S., Pobric, G., Lavidor, M., and Walsh, V. (2009). Functional representation of living and nonliving domains across the cerebral hemispheres: a combined event-related potential/transcranial magnetic stimulation study. J. Cogn. Neurosci. 21, 403-414. doi: 10.1162/jocn.2008. 21030

Gelman, A., and Stern, H. (2006). The difference between "Significant" and "Not Significant" is not itself statistically significant. Am. Stat. 60, 328-331. doi: $10.1198 / 000313006 \times 152649$

Glass, A. L., and Meany, P. J. (1978). Evidence for two kinds of lowtypical instances in a categorization task. Mem. Cogn. 6, 622-628. doi: 10.3758/BF03198252

Harpaz, Y., Levkovitz, Y., and Lavidor, M. (2009). Lexical ambiguity resolution in Wernicke's area and its right homologue. Cortex 45, 1097-1103. doi: 10.1016/j.cortex.2009.01.002

Jung-Beeman, M. (2005). Bilateral brain processes for comprehending natural language. Trends Cogn. Sci. 9, 512-518. doi: 10.1016/j.tics.2005. 09.009 times for artifactual compared to natural categories (Fuggetta et al., 2009).

More importantly, the conclusion drawn by the authors seems to be not supported by the presented results. Indeed, given their analytical approach (i.e., the use of four-way ANOVAs), to claim that the rTMS over $\mathrm{rW}$ selectively delayed responses to atypical member names, as compared to both typical and non-member names, the crucial TMS-Condition $\times$ Typicality $\times$ Membership interaction should have been significant, but this was not the case in both by-subjects and by-items full-factorial ANOVAs. Rather, this conclusion is only supported by a TMSCondition $\times$ Typicality interaction. However, it makes little sense to speak about typicality for non-member names, as typicality is a category-specific measure. Consequently, the authors made "a statistical error that is common in the neuroscience literature" (Nieuwenhuis et al., 2011, p. 1105; see also Gelman and Stern, 2006). Indeed, albeit the crucial TMS-Condition $\times$ Typicality $\times$ Membership interaction was non-significant, and without further justifications, Passeri et al. inappropriately performed separate ANOVAs. More importantly, in doing so they eliminated the sham (control) condition without justifications. Therefore, the Passeri et al.'s results should be taken with cautious and interpreted in light of the fact that their TMS-dependent effects were not modulated by the exemplar membership and were not related to an appropriate control condition for non-specific TMS effects.

To resume, Passeri et al.'s study does not permit disentangling among competing SM theories for the lack of control over some semantic/lexical dimensions of conceptual representation (Montefinese and Vinson, 2015) and of a clear semantic theoretical framework, impeding to fully appreciate their innovative contribution. However, it represents a valuable step toward creating a bridge between semantic and language theories that could resolve some of the vexing issues in both domains (Vinson et al., 2014), stimulating further research in this direction.

McRae, K., Cree, G. S., Westmacott, R., and de Sa, V. R. (1999). Further evidence for feature correlations in semantic memory. Can. J. Exp. Psychol. 53, 360-373. doi: 10.1037/h0087323

McRae, K., de Sa, V. R., and Seidenberg, M. S. (1997). On the nature and scope of featural representations of word meaning. J. Exp. Psychol. Gen. 126, 99-130. doi: 10.1037/0096-3445.126.2.99

Montefinese, M., Ambrosini, E., Fairfield, B., and Mammarella, N. (2013) Semantic memory: a feature-based analysis and new norms for Italian. Behav. Res. Methods 45, 440-461. doi: 10.3758/s13428-012-0263-4

Montefinese, M., Ambrosini, E., Fairfield, B., and Mammarella, N. (2014). Semantic significance: a new measure of feature salience. Mem. Cogn. 42, 355-369. doi: 10.3758/s13421-013-0365-y

Montefinese, M., and Vinson, D. (2015). Can the humped animal's knee conceal its name? Commentary on: "The roles of shared vs. distinctive conceptual features in lexical access.” Front. Psychol. 6:418. doi: 10.3389/fpsyg.2015.00418

Montefinese, M., Zannino, G. D., and Ambrosini, E. (2015). Semantic similarity between old and new items produces false alarms in recognition memory. Psychol Res. 79, 785-794. doi: 10.1007/s00426-014-0615-z

Nieuwenhuis, S., Forstmann, B. U., and Wagenmakers, E. J. (2011). Erroneous analyses of interactions in neuroscience: a problem of significance. Nat. Neurosci. 14, 1105-1107. doi: 10.1038/nn.2886 
Passeri, A., Capotosto, P., and Di Matteo, R. (2015). The right hemisphere contribution to semantic categorization: a TMS study. Cortex 64, 318-326. doi: 10.1016/j.cortex.2014.11.014

Rosch, E., and Mervis, C. B. (1975). Family resemblances: studies in the internal structure of categories. Cogn. Psychol. 7, 573-605. doi: 10.1016/00100285(75)90024-9

Vinson, D., Andrews, M., and Vigliocco, G. (2014). "Giving words meaning: why better models of semantics are needed in language production research" in Oxford Handbook of Language Production, eds V. Ferreira, M. Goldrick, and M. Miozzo (Oxford: Oxford University Press), 134-151.
Conflict of Interest Statement: The authors declare that the research was conducted in the absence of any commercial or financial relationships that could be construed as a potential conflict of interest.

Copyright (c) 2015 Montefinese, Ciavarro and Ambrosini. This is an open-access article distributed under the terms of the Creative Commons Attribution License (CC $B Y)$. The use, distribution or reproduction in other forums is permitted, provided the original author(s) or licensor are credited and that the original publication in this journal is cited, in accordance with accepted academic practice. No use, distribution or reproduction is permitted which does not comply with these terms. 